\title{
THE SEDIMENTATION RATE OF THE RED BLOOD CELLS IN VARIOUS TYPES OF ARTHRITIS
}

\author{
BY THEODORE W. OPPEL, WALTER K. MYERS AND \\ CHESTER S. KEEFER \\ (From the Thorndike Memorial Laboratory, Second and Fourth Medical Services (Harvard), \\ Boston City Hospital, and the Department of Medicine, Harvard Medical School, \\ Boston)
}

(Received for publication October 11, 1932)

The increased sedimentation rate of red blood cells in various diseases has aroused considerable interest during the past ten years. The positive value as an aid in differential diagnosis and in following the course of certain diseases, such as tuberculosis, has been repeatedly studied. In joint disease there is considerable divergence of opinion regarding the value of the sedimentation rate as an aid in discriminating between various types of arthritis $(1,2,4,6,7,8)$. This is probably due to the different criteria used for establishing a diagnosis, the different methods employed for determining the sedimentation rate, the stage of the disease at which the examination is made, and the presence of factors other than the fundamental disease process which alter the rate. All of these must be considered before conclusions can be drawn.

In order to determine for ourselves the value of the sedimentation rate of the red blood corpuscles in the differential diagnosis and prognosis of arthritis we studied this reaction in 107 patients with various forms of arthritis. During the same period of time 103 patients with a miscellany of diseases were studied as a control group. A list of the cases is summarized in Table I.

TABLE I

Distribution of cases charted

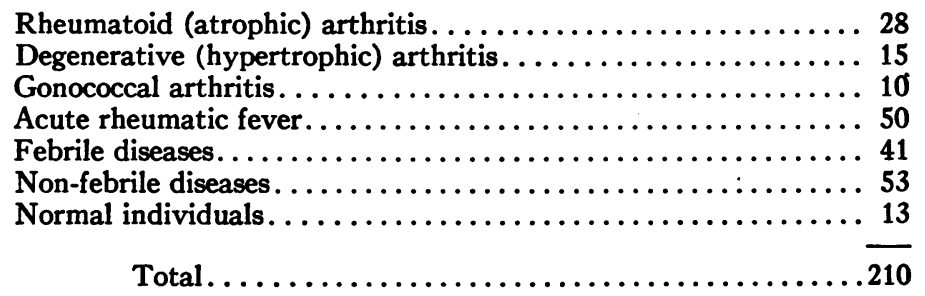

In any consideration of the value of the sedimentation rate of red blood cells, the classification of cases and the method of performing the test are of importance. 
It will be generally admitted that the precise differentiation between rheumatoid and other forms of arthritis may be difficult in many instances. It is desirable, therefore, to describe briefly the criteria used in our study. The diagnosis of rheumatoid arthritis was made on the basis of a chronic symmetrical progressive polyarthritis characterized by periarticular swelling, atrophy of the muscles in the neighborhood of the affected joints, limitation of motion due to fibrosis, destruction of the joint spaces, and atrophy of the bone. Degenerative arthritis, on the other hand, was diagnosed when one or more of the larger joints showed destruction of the articular cartilage, an increase in the density of the bone with overgrowth of the cartilage and bone at the margins of the joints, and an absence of bony ankylosis. When a patient showed some of both characteristics, the case was classified in accordance with the predominant one.

In the group of gonococcal arthritis are included the cases of arthritis accompanying gonococcal infection of the genito-urinary tract, and also the ones in which gonococci were recovered from the synovial fluid.

Acute rheumatic fever is considered with the cases of arthritis, not because it is primarily a disease of the joints, but rather a condition in which arthritis occurs frequently. There are included only cases with definite valvular disease of the heart, or myocardial changes demonstrable by electrocardiographic examination

In the control group of febrile diseases, erysipelas, acute tonsillitis, lobar pneumonia, acute respiratory infections, diabetes with infection, tuberculosis, and pleurisy are represented. Of the non-febrile diseases cases of syphilis, peptic ulcer, nephritis, diabetes, carcinoma, anemia, and rheumatic and arteriosclerotic heart diseases are included.

The method for determining the sedimentation rate is highly important. After investigating several of the techniques in common use, we concluded that the method used by Rourke and Ernstene (5) was without doubt very reliable and accurate. It is particularly satisfactory since the sedimentation rate can be corrected in accordance with the hematocrit reading, which eliminates errors due to a reduction in the number of red blood cells. It is recorded in millimeters per minute, and the normal rate when corrected varies between 0.1 and $0.3 \mathrm{~mm}$. per minute. In our work this method was adopted and followed throughout.

\section{RESULTS}

The sedimentation rate of the cases studied are recorded in Figure 1. There were great variations in the various forms of arthritis. The most rapid rates were seen in acute rheumatic fever, rheumatoid and gonococcal arthritis, and in the febrile diseases.

Patients with rheumatoid arthritis showed a more rapid sedimentation rate than the group with degenerative arthritis, but there was no striking difference between the two groups. Of the patients with other forms of 
arthritis, those with rheumatic fever showed the most rapid rates, but it is to be clearly understood from an examination of the sedimentation rate figures, that it is impossible to differentiate the cases of rheumatic fever with arthritis, from other cases of acute arthritis.

The reason for the great variation in the same disease is probably due to the changing state of the patient and to the stage of the disease when the examination is made. It has been pointed out by Ernstene (3) that as patients with acute rheumatic fever improve, the sedimentation rate decreases. We have observed this and shown it to be true also in some cases of rheumatoid arthritis. In Figures 2 and 3 are charted the sedimentation rates over periods of months of patients with rheumatic fever, and rheumatoid arthritis, respectively. In Figure 2 it is seen that there

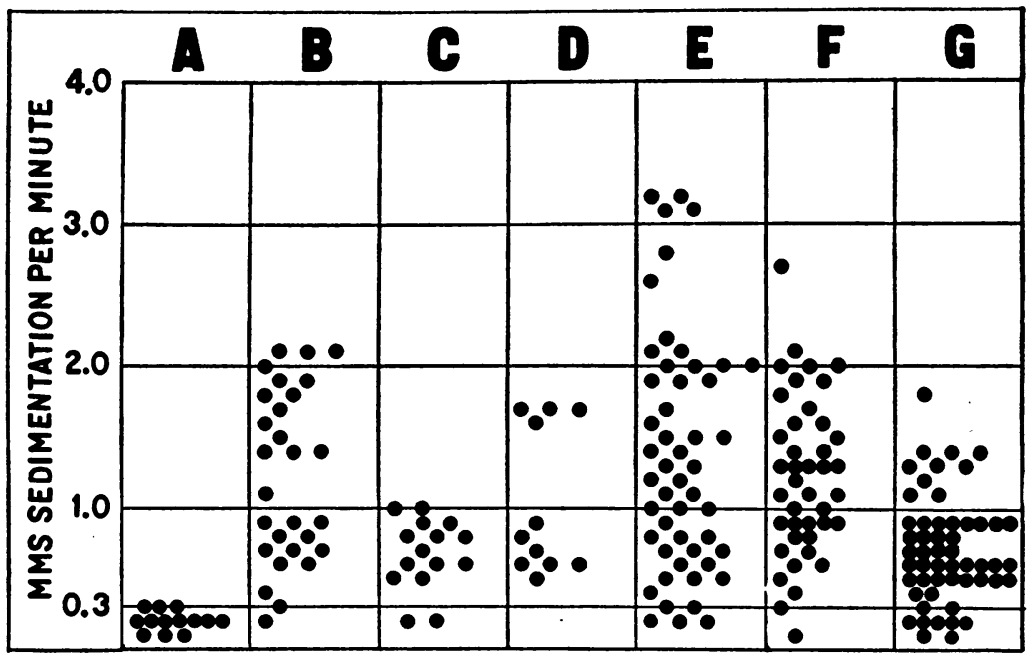

Fig. 1. The Sedimentation Rate in Various Groups of Cases

A. Normals. B. Rheumatoid arthritis. C. Degenerative arthritis. D. Gonococcal arthritis. E. Acute rheumatic fever. F. Acute febrile diseases. G. Miscellaneous non-febrile conditions.

is a tendency for the rate to fall toward normal as the patient improves. The decrease in rate is usually coincident with clinical improvement, but the latter may be observed before the former.

In the case of rheumatoid arthritis, there were variations in the rate in the same patient but they were less striking, and there was a greater tendency for the rate to remain elevated for longer periods of time. This is to be expected as the disease is chronic. In some patients $(C, D, A)$, increases in the rate were observed without any noticeable increase in the severity of the arthritis, and, in other patients, clinical improvement was not followed by a decrease in the sedimentation rate even over a period of several months. 


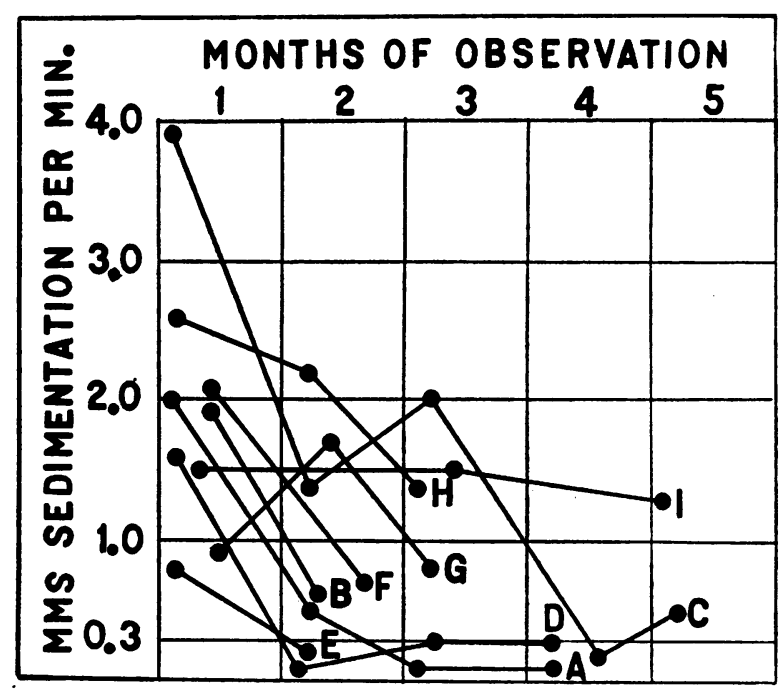

Fig. 2. Chart Showing Variation in the Corrected Sedimentation Rate in Patients with Acute Rheumatic Fever

Each letter, A-I, represents the various determinations in individual patients.

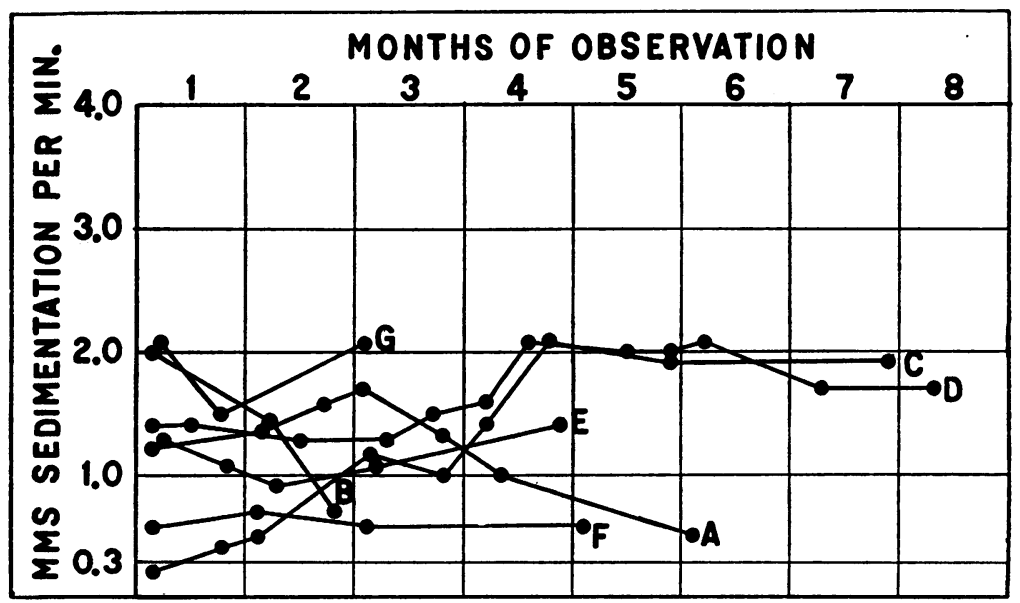

Fig. 3. Chart Showing the Variation of the Corrected Sedimentation Rate Over a Period of Months in Patients with Rheumatold Arthritis

Each letter, A-F, represents the various determinations in individual patients. 
These observations indicate that the sedimentation rate varies with the clinical course of rheumatoid arthritis, and may be of some assistance in following the patient's illness, objectively. Certain factors, however, which are quite independent of the natural course of the disease, may alter the sedimentation rate and lead to erroneous conclusions unless interpreted correctly. For example, a decided increase in the corrected sedimentation rate was observed in a patient with rheumatic fever following a hemorrhage from the larynx due to a papilloma. This increase occurred without an anemia, and without any evidence of increase in the activity of the rheumatic fever. A similar increase in the sedimentation rate was likewise observed following a profuse epistaxis in a patient with rheumatoid arthritis. It must not be forgotten that factors other than an exacerbation of the primary disease may cause an increase in the sedimentation reaction, and these must be recognized in order to interpret the results correctly.

\section{COMMENT}

Our experience indicates that isolated observations of the sedimentation rate of the red blood cells is of very doubtful value in differentiating various clinical forms of arthritis. In general, it was true that higher rates were observed in acute rheumatic fever and rheumatoid (atrophic) arthritis, than in some cases of degenerative arthritis but there were many exceptions. The wide variations observed in the different conditions studied were certainly due in part to the stage of the disease in which the observations were made. In some cases of rheumatoid arthritis and acute rheumatic fever the sinking rate of the red cells decreased as the patients improved; in others it remained elevated in spite of clinical improvement. It is possible, therefore, that repeated examinations over a period of weeks or months may give an objective indication of the progress of the underlying disease process. These changes, however, must be interpreted in the light of the entire clinical course, since factors other than the fundamental disease process can alter the sedimentation rate.

\section{SUMMARY AND CONCLUSIONS}

The sedimentation rate of the red blood cells was determined in 210 patients who were divided in to three groups, namely, patients with various forms of arthritis, patients with febrile diseases, and patients with nonfebrile diseases.

On the average, the patients with rheumatoid (atrophic) and gonococcal arthritis, and those with acute rheumatic fever, had a more rapid sedimentation rate than patients with degenerative arthritis. There were many exceptions so that as an isolated observation the test was of little value in differentiating between various forms of arthritis.

The variation in the corrected sedimentation rates in the different 
conditions studied, depended upon the stage of the disease at which the examination was made, and upon factors other than the fundamental disease process.

The test is of value in following the course of some diseases, such as acute rheumatic fever and rheumatoid (atrophic) arthritis.

\section{BIBLIOGRAPHY}

1. Dawson, M. H., Sia, R. H. P., and Boots, R. H., J. Lab. and Clin. Med., 1930, xv, 1065. The Differential Diagnosis of Rheumatoid and Osteoarthritis. The Sedimentation Reaction and Its Value.

2. Weiss, Arthur, Am. J. M. Sc., 1931, clxxxi, 379. The Prognostic Value of the Sedimentation Rate in Arthritis. A Modification of the Technique.

3. Ernstene, A. Carlton, Am. J. M. Sc., 1930, clxxx, 12. Erythrocyte Sedimentation, Plasma Fibrinogen and Leukocytosis as Indices of Rheumatic Infection.

4. Rourke, M. D., and Plass, E. D., J. Clin. Invest., 1929, vii, 365. An Investigation of Various Factors Which Affect the Sedimentation Rate of the Red Blood Cells.

5. Rourke, M. D., and Ernstene, A. Carlton, J. Clin. Invest., 1930, viii, 545. A Method for Correcting the Erythrocyte Sedimentation Rate for Variations in the Cell Volume Percentage of Blood.

6. Kahlmeter, G., Klin. Wchnschr., 1926, v, 889. Über die Bedeutung der Fahreusschen senkungsreaktion bei akuten und chronischen Arthritiden.

7. Sharpless, F. C., Atlantic M. J., 1927, xxxi, 10. Blood Sedimentation Time in Acute Rheumatic Fever.

8. Kling, D. H., Arch. Int. Med., 1932, i, 419. Sedimentation Rate of Blood Corpuscles in Synovial Fluid and in Plasma: Method of Estimation and Significance in Arthritis. 